\title{
On new "explicit formulas" in prime number theory I
} by

\section{S. KNAPOWSKI (Poznań)}

1. The distribution of prime numbers is closely bound up with the distribution of the complex zeros of the Riemann zeta-function. The following "explicit formula", due to Riemann, was first rigorously proved by von Mangoldt (see e. g. [1], p. 77, Theorem 29) and gives perhaps the most striking evidence of this connection.

If

$$
x>1, \quad \psi(x)=\sum_{n \leqslant x} \Lambda(n)
$$

where

then

$$
\begin{aligned}
A(n)= & \left\{\begin{array}{cl}
\log p & \text { if } n=p^{m}, p \text { prime, } m=1,2, \ldots, \\
0 & \text { otherwise, }
\end{array}\right. \\
& \psi_{0}(x)=\frac{1}{2}(\psi(x-0)+\psi(x+0)),
\end{aligned}
$$

$$
\psi_{0}(x)=x-\sum_{\varrho} \frac{x^{e}}{\varrho}-\frac{\zeta^{\prime}}{\zeta}(0)-\frac{1}{2} \log \left(1-\frac{1}{x^{2}}\right) ;
$$

$\varrho=\beta+i \gamma$ denoting all complex zeta-zeros and $\sum_{\varrho}\left(x^{\varrho} / \varrho\right)$ being the limit of $\sum_{|\gamma| \leqslant T^{2}}\left(x^{\varrho} / \varrho\right)$ as $T \rightarrow \infty$. Replacing the infinite series $\sum_{\varrho}\left(x^{\ell} / \varrho\right)$ by its partial sum we can deduce from (1.1) an approximate formula (see e. g. [1], p. 77, Theorem 29).

$$
\psi_{0}(x)=x-\sum_{|y| \leqslant x^{2}} x^{e}+O\left(\log ^{2} x\right) \quad \text { for } \quad x \geqslant 2 .
$$

Formula (1.1) suggest at once the well-known fact that the error $\max _{1 \leqslant x \leqslant T}\left|\psi_{0}(x)-x\right|$ in the prime number formula lies in the interval $\left\langle T^{\theta-8}, T^{\theta+s}\right\rangle$, for large $T, \theta$ being the upper bound of the real parts of all zeta-zeros. This illustrates the significance of Riemann's conjecture $\theta=\frac{1}{2}$. 
It was Turán who recognized that it is of interest in the prime number theory not only to investigate the zeta-zeros but also the zeros of

$$
U_{N}(S)=\sum_{n \leqslant N} \frac{1}{n^{8}} .
$$

He has proved the following theorem:

If for integer $N>n_{0}$ the partial-sums $U_{N}(s)$ do not vanish in the half-plane

$$
\sigma>1+\frac{\log ^{100} N}{\sqrt{N}}, \quad s=\sigma+i t,
$$

then Riemann's hypothesis is true $\left.{ }^{1}\right)$.

In this paper I prove a new "explicit formula", similar to (1.L) but depending on the zeros of $U_{N}(s)$ instead of the zeta-zeros.

The result is as follows:

For $2 \leqslant x \leqslant N, N \geqslant N_{0}$, we have

$$
\psi_{0}(x)=\frac{\log N !}{N}-\sum_{\varrho} \frac{x^{\varrho}}{\varrho} ;
$$

$\varrho=\beta+i \gamma$ denoting the zeros $U_{N}(s)$ and $\sum_{Q}\left(x^{Q} / \varrho\right)$ being the limit of $\sum_{|y| \leqslant T^{2}}\left(x^{\ell} / \varrho\right)$ as $T \rightarrow \infty\left({ }^{2}\right)$.

Further I prove some estimates for $\sum_{|\gamma|>T}\left(x^{\varrho} / \varrho\right)$, which give information about the behaviour of the series $\sum_{e}\left(x^{\varrho} \mid \varrho\right)$ analogous to that in the classicall case.

The formula (1.2) implies the following corollary, which gives a new approximation for the "remainder term" $\psi_{0}(x)-x$ :

If $x \geqslant 2$ then

$$
\psi_{0}(x)=x-\sum_{\substack{\beta \geqslant-1 \\|\gamma| \leqslant x^{16}}} \frac{x^{2}}{\varrho}+O(\log x),
$$

$\varrho=\beta+i \gamma$ being the zeros of $U_{N}(S)$ for $N=\left[e^{x}\right]\left({ }^{3}\right)$.

(1) See [4], p. 4. The quoted rosult is an oasy combination of thooroms If and if of the paper.

$\left(^{2}\right) \sum_{T}\left(x^{\varrho} / \varrho\right)$ could be understood as $\lim \underset{ }{\sum}\left(x^{0} / \varrho\right)$, but it is not nocessary in virtue of a cortain theorem of Polya (see [2]) whieh impliss that there in ouly a finite number of $U_{N}(s)$-zeros in evory strip $-\infty<A \leqslant t \leqslant B<+\infty$.

$\left(^{3}\right)[\alpha]$ denotes as usual the integral part of $\alpha$.
I wish to express my deep gratitude to Professor Paul Turán, who has made a number of important suggestions.

2. LEMMA 1. (a) The number of zeros of $U_{N}(s)$ in the rectangle

$$
0 \leqslant \sigma \leqslant 2, \quad n \leqslant t \leqslant n+1, \quad s=\sigma+i t \quad(n=0,1,2, \ldots)
$$

is $\leqslant c_{1} \log N\left(^{4}\right)$.

(b) The number of zeros of $U_{N}(s)$ in the rectangle

$$
-m \leqslant \sigma \leqslant-m+1, \quad n \leqslant t \leqslant n+1, \quad s=\sigma+i t
$$

$(n=0,1,2, \ldots, m=1,2, \ldots)$ is $\leqslant c_{2} m \log N$.

Proof. Apply Jensen's inequality (see e. g. [1], p. 49, Theorem D). The number of zeros of $f(s)$ in the circle $\left|s-s_{0}\right|<R$ is

$$
\leqslant \log \max _{\left|s-s_{0}\right|=R e}\left|\frac{f(s)}{f\left(s_{0}\right)}\right| .
$$

Put, for (a), $s_{0}=2+\left(n+\frac{1}{2}\right) i, R=\sqrt{5}$. Then

$$
U_{N}(s)=O\left(N^{\sqrt{5}-1}\right), \quad\left|U_{N}\left(s_{0}\right)\right| \geqslant 2-\frac{1}{6} \pi^{2}>0
$$

and

$$
\log _{\left|\delta-s_{0}\right|=R e}\left|\frac{U_{N}(s)}{U_{N}\left(s_{0}\right)}\right| \leqslant c_{1} \log N .
$$

For (b) put $s_{0}=2+\left(n+\frac{1}{2}\right) i, R=\sqrt{(m+2)^{2}+1}$, whence

and

$$
U_{N}(s)=O\left(N^{R e-1}\right)
$$

$$
\log \max _{\left|s-s_{0}\right|=R e}\left|\frac{U_{N}(s)}{U_{N}\left(s_{0}\right)}\right| \leqslant c_{2} \log N .
$$

LEMMA 2. (a) In the rectangle

$$
-\frac{1}{2} \leqslant \sigma \leqslant 2, \quad n \leqslant t \leqslant n+1, \quad s=\sigma+i t \quad(n=0,1,2, \ldots)
$$

we have

$$
\left|\frac{U_{N}^{\prime}}{\bar{U}_{N}}(s)-\sum_{\varrho} \frac{1}{s-\varrho}\right| \leqslant c_{3} \log N,
$$

where $\varrho$ runs through the zeros of $U_{N}(s)$ lying in the rectangle

$$
-1 \leqslant \sigma \leqslant 2, \quad n-\frac{1}{2} \leqslant t \leqslant n+\frac{3}{2}, \quad s=\sigma+i t .
$$

${ }^{4}$ ) Throughout this paper $c_{1}, c_{2}, \ldots$ always denote positive numerical constants. 
(b) In the rectangle

$$
-m-\frac{1}{2} \leqslant \sigma \leqslant-m+1, \quad n \leqslant t \leqslant n+1, \quad s=\sigma+i t
$$

$(n=0,1,2, \ldots ; m=1,2, \ldots)$ we have

$$
\left|\frac{U_{N}^{\prime}}{U_{N}}(s)-\sum_{\varrho} \frac{1}{s-\varrho}\right| \leqslant c_{4} m \log N
$$

where $\varrho$ runs through the zeros of $U_{N}(s)$ lying in the rectangle

$$
-m-1 \leqslant \sigma \leqslant-m+2, \quad n-\frac{1}{2} \leqslant t \leqslant n+\frac{3}{2}, \quad s=\sigma+i t .
$$

Proof. I will prove only (b).

Put $s_{0}=2+\left(n+\frac{1}{2}\right) i$ and consider the function $G(z)=U_{N}\left(z+s_{0}\right)$ in the circle $|z| \leqslant 2 R, R=\sqrt{1+(m+3)^{2}}$. Denote by $z_{k}$ all the zeros of $G(z)$ in the circle $|z|<\frac{3}{2} R$. The function

$$
G(z)\left(\prod_{z_{k}} 2 R \frac{z-z_{k}}{4 R^{2}-z \bar{z}_{k}}\right)^{-1} \equiv G_{1}(z)
$$

is regular and $\neq 0$ in the circle $|z|<\frac{3}{2} R$. We showed above that

$$
\left|\frac{G(z)}{G(0)}\right| \leqslant \exp \left(c_{5} m \log N\right) \quad \text { if } \quad|z| \leqslant 2 R .
$$

Denote by $G_{2}(z)$ the branch of $\log \left(G_{1}(z) / G_{1}(0)\right)$ in the circle $|z|<\frac{3}{2} R$, determined by $G_{2}(0)=0$. We have for $|z|=2 R$

$$
\left|\frac{G_{1}(z)}{G_{1}(0)}\right| \leqslant\left|\frac{G(z)}{G(0)}\right| \leqslant \exp \left(c_{5} m \log N\right) \quad \text { (compare e. g. [1], p. 49). }
$$

Hence

and

$$
\Re G_{2}(z) \leqslant c_{5} m \log N \quad \text { for } \quad|z|<\frac{3}{2} R
$$

$$
\left|\frac{G_{1}^{\prime}}{G_{1}}(z)\right| \leqslant \frac{2 \cdot \frac{3}{2} R}{\left(\frac{1}{2} R\right)^{2}} c_{5} m \log N \leqslant c_{6} \log N \quad \text { for } \quad|z| \leqslant R
$$

(apply [1], p. 50, Theorem $\mathbf{E}$ with $v=1$ ). That is

$$
\left|\frac{G^{\prime}}{G}(z)-\sum_{z_{k}}\left(\frac{1}{z-z_{k}}+\frac{1}{4 R^{2} / \bar{z}_{k}-z}\right)\right| \leqslant c_{6} \log N \quad \text { for } \quad|z| \leqslant R .
$$

But

$$
\left|\frac{4 R^{2}}{\bar{z}_{k}}-z\right| \geqslant \frac{4 R^{2}}{\frac{3}{2} R}-R=\frac{5}{3} R
$$

and the number of the zeros $z_{k}$ is $\leqslant c_{7} m \log N$, whence

Finally

$$
\left|\sum_{z_{k}} \frac{1}{4 R^{2} / \bar{z}_{k}-z}\right| \leqslant \frac{3}{5 R} c_{7} m \log N \leqslant c_{8} \log N \text {. }
$$

$$
\left|\frac{U_{N}^{\prime}}{U_{N}}(s)-\sum_{s_{k}} \frac{1}{s-s_{k}}\right| \leqslant c_{9} \log N
$$

after the transformation $s=z+s_{0}$. If $s_{k}$ lies outside the rectangle

$$
-m-1 \leqslant \sigma \leqslant-m+2, \quad n-\frac{1}{2} \leqslant t \leqslant n+\frac{3}{2}, \quad s=\sigma+i t,
$$

then $\left|s-s_{k}\right| \geqslant \frac{1}{2}$ for

$$
-m-\frac{1}{2} \leqslant \sigma \leqslant-m+\frac{3}{2}, \quad n \leqslant t \leqslant n+1, \quad s=\sigma+i t
$$

and the result follows.

LEMTMa 3.

(a) There exists a sequence of numbers $T_{0}, T_{1}, T_{2}, \ldots$ such that

1. $n \leqslant T_{n} \leqslant n+1$

2. $\left|\frac{U_{N}^{\prime}}{U_{N}}(s)\right| \leqslant c_{10} \log ^{2} N$ for $-\frac{1}{2} \leqslant \sigma \leqslant 2, t=T_{n}, s=\sigma+i t$.

(b) For every $m=1,2, \ldots$ there exists a sequence $T_{0}^{(m)}, T_{1}^{(m)}, T_{2}^{(m)}, \ldots$ such that

1. $n \leqslant T_{n}^{(m)} \leqslant n+1$,

2. $\left|\frac{U_{N}^{\prime}}{U_{N}}(s)\right| \leqslant c_{11} m^{2} \log ^{2} N$ for $-m-\frac{1}{2} \leqslant \sigma \leqslant-m+1, t=T_{n}^{(m)}$,

$$
s=\sigma+i t
$$

(c) For every $m=1,2, \ldots$ there exists a sequence $S_{0}^{(m)}, S_{1}^{(m)}, S_{2}^{(m)}, \ldots$ such that

1. $-m+\frac{1}{2} \leqslant S_{n}^{(m)} \leqslant-m+1$,

2. $\left|\frac{U_{N}^{\prime}}{U_{N}}(s)\right| \leqslant c_{12} m^{2} \log ^{2} N$ for $\sigma=S_{n}^{(m)}, n \leqslant t \leqslant n+1, s=\sigma+i t$.

(d) For every $m=1,2, \ldots$ there exists a sequence $\tilde{S}_{0}^{(m)}, \tilde{S}_{1}^{(m)}, \tilde{S}_{2}^{(m)}, \ldots$ such that

1. $-m \leqslant \tilde{\mathcal{S}}_{n}^{(m)} \leqslant-m+1$, 
2. $\left|\frac{{U_{N}^{\prime}}^{*}}{U_{N}}(s)\right| \leqslant c_{13} m^{2} \log ^{2} N$ for $\sigma=\tilde{S}_{n}^{(m)}, n \leqslant t \leqslant n+\frac{3}{2}, s=\sigma+i t$.

(e) For every $m=1,2, \ldots$ there exists a sequence $\tilde{T}_{0}^{(m)}, \tilde{T}_{1}^{(m)}, \tilde{T}_{2}^{(m)}, \ldots$ such that

1. $n \leqslant \tilde{T}_{n}^{(m)} \leqslant n+\frac{1}{2}$

2. $\left|\frac{U_{N}^{\prime}}{U_{N}}(s)\right| \leqslant c_{14} m^{2} \log ^{2} N$ for $-m \leqslant \sigma \leqslant-m+1, t=\tilde{T}_{n}^{(m)}$,

$$
s=\sigma+i t
$$

Proof. Since all the proofs are analogous, it is clearly enough to prove, say, (b).

Divide the interval $\langle n, n+1\rangle$ into $Q+1$ equal parts, where $Q$ denotes the number of zeros of $U_{N}(s)$ in the rectangle

$$
-m-\frac{1}{2} \leqslant \sigma \leqslant-m+1, \quad n \leqslant t \leqslant n+1, \quad s=\sigma+i t .
$$

At least one of the rectangles so obtained is tree of $U_{N}(s)$-zeros. Denote the ordinate of the centre of this rectangle by $T_{n}^{(m)}$. Thet $\varrho$ be any $U_{N}(s)$ zero lying in the rectangle

$$
-m-1 \leqslant \sigma \leqslant-m+2, \quad n-\frac{1}{2} \leqslant t \leqslant n+\frac{3}{2}, \quad s=\sigma+i t
$$

and let $s^{*}$ lie on the line $t=T_{n}^{(m)}$. Then

$$
\left|s^{*}-\varrho\right| \geqslant \frac{1}{2} \cdot \frac{1}{Q+1},
$$

whence

$$
\frac{1}{\left|s^{*}-\varrho\right|} \leqslant 2(Q+1) \leqslant c_{15} m \log N
$$

by Lemma 1. Hence and from Lemma 2 we obtain

for

$$
\left|\frac{U_{N}^{\prime}}{U_{N}}(s)\right| \leqslant c_{11} m^{2} \log ^{2} N
$$

$$
-m-\frac{1}{2} \leqslant \sigma \leqslant-m+1, \quad t=T_{n}^{(m)}, \quad s^{\prime}=\sigma+i t .
$$

Lamma 4. The funation $U_{N}^{\prime}(s) / U_{N}(s)$ may be developed in a Diriohlet series $\sum_{n=1}^{\infty}\left(\alpha_{n} / n^{s}\right)$ convergent in the half-plane

$$
\sigma>1+2 \frac{\log \log N}{\log N} \quad\left(N \geqslant N_{0}\right) .
$$

\section{Further}

and

$$
a_{n}=-\Lambda(n) \quad \text { for } \quad n \leqslant N
$$

(2.2) if $x \leqslant N$ and $n \leqslant \frac{3}{2} x$ then $\left|\alpha_{n}\right| \leqslant c_{16} x \log x$.

Remark. It is sufficient to prove (2.2) only for $x=N$. In fact, if $x \leqslant \frac{2}{3} N$, then $n \leqslant N$ and $\left|a_{n}\right| \leqslant \log n \leqslant \log \frac{3}{2} x$ by (2.1). If $\frac{2}{3} N \leqslant x \leqslant N$, then $\left|\alpha_{n}\right| \leqslant c_{16} N \log N \leqslant c_{16}^{\prime} x \log x$, since we suppose that (2.2) holds for $x=N$.

Proof. The existence of a development of $U_{N}^{\prime}(s) / U_{N}(s)$ in a Dirichlet series follows from Turán's remark (10.1.7) in [5], p. 121. The convergence of this series for

$$
\sigma>1+2 \frac{\log \log N}{\log N}
$$

follows from another theorem of Turán ( $[4]$, p. 20), stating that

$$
\left|\frac{1}{U_{N}(s)}\right| \leqslant c_{17} \frac{\log ^{2} N}{(\log \log N)^{2}} \quad \text { for } \quad \sigma>1+2 \frac{\log \log N}{\log N} .
$$

This inequality, combined with a general theorem on Dirichlet series (see e. g. [3], §9.44, p. 302), gives the required result. We note incidentally that (2.3) implies the inequality

(2.4) $\quad\left|\frac{U_{N}^{\prime}}{U_{N}}(s)\right| \leqslant o_{18} \frac{\log ^{4} N}{(\log \log N)^{3}}$. for $\quad \sigma>1+2 \frac{\log \log N}{\log N}$,

which we will use afterwards. Turning now to the proof of (2.1) we have

$$
U_{N}^{\prime}(s)=U_{N}(s) \sum_{n=1}^{\infty} \frac{a_{n}}{n^{s}}
$$

Hence, for $n \leqslant N$ we obtain

$$
\sum_{k \mid n} \alpha_{k}=-\log n
$$

whence by the Möbius inversion formula we obtain

$$
a_{n}=\sum_{d \mid n} \mu(d)(-\log (n / d))
$$

Since this defines $\alpha_{n}$ uniquely for $n \leqslant N$, from the well-known formula

$$
\sum_{n=1}^{\infty} \frac{-\Lambda(n)}{n^{s}}=\frac{\zeta^{\prime}(s)}{\zeta(s)}=\sum_{n=1}^{\infty} \frac{-\log n}{n^{s}} \cdot \sum_{n=1}^{\infty} \frac{\mu(n)}{n^{s}} \quad \text { for } \quad \sigma>1
$$

we see at once that (2.1) holds. 
Now we shall prove inequality (2.2) for $x=N$. It is sufficient to consider only $N<n \leqslant \frac{3}{2} N$. We then have

$$
\sum_{k \mid n} \alpha_{k}=0 .
$$

If $k<n$, then $k \leqslant \frac{1}{2} n$ and consequently $z_{0} \leqslant N$. Hence

$$
a_{n}=-\sum_{\substack{k, \mid n \\ k<n}} a_{k}, \quad\left|a_{n}\right| \leqslant \sum_{\substack{k, n \\ k<n}}\left|\alpha_{k}\right| \leqslant \sum_{k_{k \mid} \mid n}^{\top} \log k \leqslant \log n ! \leqslant c_{16} N \log N .
$$

3. THEOREM. Let $N>N_{0}$ be an integer. If $2 \leqslant x \leqslant N$, then

$$
\psi_{0}(x)=\frac{\log N !}{N}-\sum_{\varrho} \frac{x^{\ell}}{\varrho},
$$

$\varrho=\beta+i \gamma$ running through the zeros of $U_{N}(s)=\sum_{n \leqslant N}\left(1 / n^{s}\right)$, and $\sum_{Q}\left(x^{\varrho} / \varrho\right)$ $=S_{N}(x)$ denoting the limit of $S_{N}(x, T)=\sum_{|\gamma| \leqslant T}\left(x^{\ell} / \varrho\right)$ as $T \rightarrow \infty$.

\section{Writing further}

we have

$$
R_{N}(x, T)=S_{N}(x)-S_{N}(x, T)
$$

$$
\begin{aligned}
& \left|R_{N}(x, T)\right| \leqslant \begin{cases}c_{19} \frac{x^{2}}{T}\left(\frac{\log ^{14} N}{(\log \log N)^{6}}+\frac{\log ^{6} N}{\xi}\right) & \text { if } \quad \text { if } ;: p^{m}, \\
c_{19} \frac{x^{2}}{T} \cdot \frac{\log ^{14} N}{(\log \log N)^{6}} & \text { if } x=p^{m},\end{cases} \\
& \left|R_{N}(x, T)\right| \leqslant c_{19}\left(\frac{x^{2}}{T} \cdot \frac{\log ^{14} N}{(\log \log N)^{6}}+\log x\right) \quad \text { always }
\end{aligned}
$$

where $\xi=\xi(x)$ is the distance of $x$ from the nearest prime power $p^{m}$.

Proof. Let $T \geqslant 3$ and denote by $T^{\prime}$ the lenst $T_{n}$ of Lemma 3 which is greater than $T$. Let $q \geqslant 2$ be any integer.

Denote by $C_{q}^{T}$ the contour consisting of the segment

$$
\left\langle 2+6 \frac{\log \log N}{\log N}-i T^{\prime \prime}, 2+6 \frac{\log \log N}{\log N}+i T^{\prime}\right\rangle
$$

and of three broken lines given by Lemma 3 as follows:
First there is given an infinite broken line consisting of the horizontal segments

$$
\begin{gathered}
S_{n}^{(1)} \leqslant \sigma \leqslant 2+6 \frac{\log \log N}{\log N}, \quad t=T_{n} . \\
S_{n}^{(m+1)} \leqslant \sigma \leqslant S_{n}^{(m)}, \quad t=T_{n}^{(m)} \quad(m=1,2, \ldots)
\end{gathered}
$$

joined by vertical ones. Secondly there is an infinite broken line consisting of the vertical segments

$$
\sigma=\tilde{S}_{\mu}^{(q)}, \quad \tilde{T}_{\mu}^{(q)} \leqslant t \leqslant \tilde{T}_{\mu+1}^{(q)} \quad(\mu=0,1, \ldots)
$$

joined by horizontal ones. By symmetrical mapping in the half-plane $t<0$ we obtain three infinite broken lines. The contour $O_{q}^{T}$ is formed by their intersection.

$$
\text { . } a=2+6 \frac{\log \log N}{\log N}-i T^{\prime}, \quad b=2+6 \frac{\log \log N}{\log N}+i T^{\prime} .
$$

Consider the integral

(3.1) $\frac{1}{2 \pi i} \int_{C_{q}^{T}} \frac{x^{s}}{s}\left(-\frac{U_{N}^{\prime}}{U_{N}}(s)\right) d s=\frac{1}{2 \pi i} \int_{a}^{b} \frac{x^{8}}{s}\left(-\frac{U_{N}^{\prime}}{U_{N}}(s)\right) d s+\frac{1}{2 \pi i}\left(I_{1}+I_{2}+I_{3}\right)$ where $I_{1}, I_{2}, I_{3}$ denote the integrals along respective upper, lower and left broken line. First of all, estimate these integrals:

(3.2) $\left|I_{3}\right| \leqslant x^{-q+1} c_{20} q^{2} \log ^{2} N\left(\int_{0}^{T^{\prime}} \frac{d t}{\sqrt{(q-1)^{2}+t^{2}}}+\sum_{n \leqslant T} \frac{1}{n}\right) \leqslant c_{21} \frac{q^{2} \log ^{2} N}{x^{q-1}} \log T$,

(3.3) $\left|I_{1}\right| \leqslant c_{22}\left(\sum_{\nu=1}^{q} \int_{\nu}^{\nu} \frac{x^{-\sigma}}{\sqrt{\sigma^{2}+(T-1)^{2}}} \nu^{2} \log ^{2} N d \sigma+\frac{x^{2} \log ^{8} N}{T}+\sum_{\nu=1}^{q} \frac{\nu^{2} \log ^{2} N}{x^{\nu-1} T}\right)$

$$
\leqslant c_{23} \frac{\log ^{8} N}{T}\left(x^{2}+\sum_{p=1}^{\infty} \frac{\nu^{2}}{2^{p-1}}\right) \leqslant c_{24} \frac{x^{2} \log ^{8} N}{T}
$$

and similarly:

$$
\left|I_{2}\right| \leqslant c_{24} \frac{x^{2} \log ^{8} N}{T}
$$

Now apply Cauchy's theorem

(3.5) $\quad \frac{1}{2 \pi i} \int_{\sigma_{q}^{T}} \frac{x^{s}}{s}\left(-\frac{U_{N}^{\prime}}{U_{N}}(s)\right) d s=\frac{\log N !}{N}-\sum_{\text {enside } C_{q}^{T}} \frac{x^{\ell}}{\varrho}$. 
As $q \rightarrow \infty$, we obtain from (3.1), (3.2), (3.3), (3.4), (3.5)

(3.6) $\frac{1}{2 \pi i} \int_{a}^{b} \frac{x^{5}}{5}\left(-\frac{U_{N}^{\prime}}{U_{N}}(s)\right) d s=\frac{\log N !}{N}-\sum_{\varrho}^{\prime} \frac{x^{Q}}{\varrho}+O\left(\frac{x^{2}}{T} \log ^{8} N\right)$

where $\Sigma^{\prime}$ denotes the summation over all zeros @ lying between the upper and the lower broken line. Now

$$
\left|\sum_{Q}^{\prime} \frac{x^{Q}}{\varrho}-S_{N}(x, T)\right| \leqslant \frac{c_{25}}{T}\left(x \log ^{3} N+\sum_{\nu=1}^{\infty} \frac{\nu \log N}{x^{p-1}}\right) \leqslant \frac{e_{26}}{T} x \log ^{3} N
$$

From this and from (3.6) we obtain

(3.7) $\frac{1}{2 \pi i} \int_{a}^{b} \frac{x^{8}}{s}\left(-\frac{D_{N}^{\prime}}{U_{N}}(s)\right) d s=\frac{\log N !}{N}-S_{N}(x, T)+O\left(\frac{x^{2}}{T} \log ^{8} N\right)$.

Now put

$I(y)=\frac{1}{2 \pi \mathrm{i}} \int_{c-i \infty}^{c+i \infty} \frac{y^{s}}{s} d s, \quad I(y, T)=\int_{c-i T}^{c+i T} \frac{y^{s}}{s} d s, \quad \Delta(y, T)=I(y)-I(y, T)$.

Then it is well known (see e.g. [1], p. 75, Theorem G) that for $T>0$

$$
\begin{aligned}
& |\Delta(y, T)|<\left\{\begin{array}{lll}
\frac{y^{c}}{\pi T|\log y|} & \text { if } & y \neq 1 \\
\frac{c}{\pi T} & \text { if } & y=1
\end{array}\right. \\
& |\Delta(y, T)|<y^{c} \quad \text { always. }
\end{aligned}
$$

Using this notation and putting $e=2+6 \frac{\log \log N}{\log N}$ we have

$$
\begin{aligned}
\frac{1}{2 \pi i} \int_{c-i T^{\prime}}^{c+i T^{\prime \prime}} \frac{x^{s}}{s}\left(-\frac{U_{N}^{\prime}}{U_{N}}(s)\right) d s & =\sum_{n=1}^{\infty} \frac{-\alpha_{n}}{2 \pi i} \int_{c-i T^{\prime \prime}}^{c+i T^{\prime}} \frac{(x / n)^{s}}{s} d s=\sum_{n=1}^{\infty}-\alpha_{n} I\left(\begin{array}{l}
\infty \\
n
\end{array}, T^{\prime \prime}\right) \\
& =\sum_{n=1}^{\infty}-a_{n} I\left(\frac{x}{n}\right)+\sum_{n=1}^{\infty} \alpha_{n} \Delta\left(\frac{\infty}{n}, T^{\prime}\right) \\
& =\psi_{0}(x)+\sum_{n=1}^{\infty} \alpha_{n} \Delta\left(\frac{x}{n}, T^{\prime}\right) .
\end{aligned}
$$

Write

Then

$$
\begin{gathered}
u_{n}=a_{n} \Delta\left(\frac{x}{n}, T^{\prime}\right), \quad \sum_{n=1}^{\infty} u_{n}=X . \\
\frac{1}{2 \pi i} \int_{a}^{b} \frac{x^{s}}{s}\left(-\frac{U_{N}^{\prime}}{\bar{U}_{N}}(s)\right) d s=\psi_{0}(x)+X,
\end{gathered}
$$

where

$$
a=2+6 \frac{\log \log N}{\log N}-i T^{\prime}, \quad b=2+6 \frac{\log \log N}{\log N}+i T^{\prime} .
$$

We now estimate $X$. Let

If $n \neq x$ then

$$
2+6 \frac{\log \log N}{\log N}=\not
$$

$$
\left|u_{n}\right| \leqslant\left|a_{n}\right| \cdot \frac{(x / n)^{d}}{\pi T^{\prime}|\log (x / n)|} \leqslant \frac{x^{2} \log ^{6} N \cdot\left|a_{n}\right|}{\pi T n^{d}} \cdot \frac{n+x}{|n-x|} .
$$

Denote by $v=v(x)$ the integer defined by $v-\frac{1}{2}<x \leqslant v+\frac{1}{2}$. Then it is not difficult to see that $\left|a_{v}\right| \leqslant \log v$ and we obtain analogously to [1] (p. 79)

$$
\begin{aligned}
& \left|u_{v}\right| \leqslant\left\{\begin{array}{lll}
c_{27} \frac{x^{2}}{T \xi} \log ^{6} N & \text { if } & x \neq p^{m}, \\
e_{28} \frac{\log x}{T} & \text { if } & x=p^{m}
\end{array}\right. \\
& \left|u_{v}\right| \leqslant c_{29} \log x \quad \text { always. }
\end{aligned}
$$

Further (compare [1], p. 79)

$$
\left|X-u_{v}\right| \leqslant \frac{x^{2} \log ^{6} N}{\pi T}\left(5 \sum_{n=1}^{\infty} \frac{\left|\alpha_{n}\right|}{n^{\bar{d}}+2} \sum_{r=1}^{[x]} \frac{\max _{1 \leqslant n \leqslant 3 x / 2}\left|a_{n}\right| \cdot \frac{5}{2} x}{\left(\frac{1}{2} x\right)^{2} \frac{1}{2} r}\right) .
$$

Clearly

$$
\sum_{n=1}^{\infty} \frac{\left|a_{n}\right|}{n^{\bar{d}}} \leqslant \sum_{n=1}^{\infty} \frac{\left|a_{n}\right|^{2}}{n^{\bar{d}}}+\sum_{n=1}^{\infty} \frac{1}{n^{\bar{d}}} .
$$

By the mean-value theorem for Dirichlet series (see e.g. [5], p. 307) and inequality (2.4) we obtain

(3.12) $\sum_{n=1}^{\infty} \frac{\left|a_{n}\right|^{2}}{n^{d}} \leqslant c_{30} \frac{\log ^{8} N}{(\log \log N)^{6}} \quad$ where $\quad d=2+6 \frac{\log \log N}{\log N}$. 
Further, by (2.2),

$$
\sum_{r=1}^{[x]} \frac{\max _{1 \leqslant n \leqslant 3 x / 2}\left|\alpha_{n}\right| \cdot \frac{5}{2} x}{\left(\frac{1}{2} x\right)^{2} \frac{1}{2} r} \leqslant c_{31} \frac{x^{2} \log x}{x^{2}} \sum_{r=1}^{[x]} \frac{1}{r} \leqslant c_{32} \log ^{2} x .
$$

(3.10), (3.11), (3.12), and (3.13) give

$$
\left|X-u_{v}\right| \leqslant c_{33} \frac{x^{2} \log ^{14} N}{T(\log \log N)^{6}} .
$$

(3.7) and (3.8) give

$$
\psi_{0}(x)=\frac{\log N !}{N}-S_{n}(x, T)+O\left(\frac{x^{2}}{T} \log ^{8} N\right)+O(|X|) .
$$

By (3.9) and (3.14) we obtain the required estimates for $R_{N}(x, T)$. The formula

$$
\psi_{0}(x)=\frac{\log N !}{N}-\sum_{\ell} \frac{x^{Q}}{\varrho}
$$

follows on letting $T$ tend to infinity.

CoroLluary. Let $x \geqslant 2$. Then

$$
\psi_{0}(x)=x-\sum_{\substack{|\gamma| \leqslant x 16 \\ \beta>-1}}^{Y} x^{\rho}+O(\log x)
$$

where $\varrho=\beta+i \gamma$ denote the zeros of $U_{n}(s)=\sum_{n \in N} \frac{1}{n^{8}}$ and $N=\left[e^{x}\right]$.

Obviously

$$
\frac{\log N !}{N}=x+O(1)
$$

\section{Taking further}

we obtain

$$
T=x^{2} \log ^{16} N
$$

$$
\begin{aligned}
\left|\sum_{\substack{|\gamma| \leqslant T \\
\mid<\beta<-m+1}} \frac{x^{e}}{\varrho}\right| & \leqslant c_{34} x^{-m+1} \sum_{n \leqslant x^{2} \log ^{14} N} \frac{m \log N}{n} \\
& \leqslant c_{35} x^{-m+1} m \log \left(x^{2} \log ^{14} N\right) \log N \leqslant o_{36} m \frac{\log x}{x^{m-2}}
\end{aligned}
$$

whence

$$
\left|\sum_{\substack{|\gamma| \leqslant T \\ p<-1}} \frac{x^{\theta}}{\varrho}\right| \leqslant c_{37} \log x \sum_{m=2}^{\infty} \frac{m}{x^{m-2}} \leqslant c_{37} \log x \sum_{m=2}^{\infty} \frac{m}{2^{m-2}}=O(\log x)
$$

and the result follows.

Remark. It is not difficult to replace $x^{16}$ in formula (3.15) by $x^{4+\varepsilon}$. This could have been achieved by taking

instead of

$$
a=2+6 \frac{\log \log x}{\log x}
$$

$$
c=2+6 \frac{\log \log N}{\log N}
$$

in the proof of Theorem and by more careful estimation.

4. Considering now the function

$$
\frac{x^{s}}{s^{k+1}}\left(-\frac{U_{N}^{\prime}}{U_{N}}(s)\right), \quad k \geqslant 1 \text { integer, }
$$

instead of

$$
\frac{x^{s}}{s}\left(-\frac{U_{N}^{\prime}}{U_{N}}(s)\right)
$$

we can obtain formulas involving $\sum_{e} \frac{x^{e}}{\varrho^{k+1}}$ analogous to those already obtained.

Put, say, $x=N$. Then we prove

(4.1) $\sum_{n \leqslant N} \Lambda(n) \frac{\log ^{k}(N / n)}{k !}=A_{k}(N)-\sum_{|\gamma| \leqslant T} \frac{N^{e}}{e^{k+1}}+O\left(\frac{N^{2}}{T^{k}} \cdot \frac{\log ^{14} N}{(\log \log N)^{6}}\right)$

where $\varrho=\beta+i \gamma$ denotes the zeros of $\sigma_{N}(s)$ and

$$
A_{k}(N)=\frac{1}{k !} \cdot \frac{d^{k}}{d s^{k}}\left(-N^{8} \frac{U_{N}^{\prime}}{U_{N}}(s)\right)_{s=0} .
$$

It can be noticed that we are in a position to deduce from (4.1) some information on the distribution of zeros of $U_{N}(s)$. As this subject seems to be of self-contained interest I will return to it somewhere else. 
[1] A. E. Ingham, The distribution of prime numbers, Cambridge 1932.

[2] N O breshloff, Lösung der Aufgabe 24. Nullstellen linearer kombinatio. nen von Exponentialfunktionen, Jahresbericht der Deutsehen Mathematiker-Vereinigung 37 (1928), Aufgaben und Lösungen, p. $82-84$.

[3] E. C. Titchmarsh, Theory of funetions, Oxford 1939.

[4] P. Turán, On some approximative Diriehlet-polynomials in the theory of the zela-junction of Riemann, Danske Vid. Selsk. Mat.-Fys. Modd. 24 no 17 (1948), p. 1 -36.

[5] - Eine neue Methode in der Analysis und deren Anwendungen, Budlupest 1953.

Regu par la Rédation le 2\%. 1. 1958.

\section{On the distribution of the solutions of diophantine equations with many unknowns}

by

I. VIIDINGER (Budapest)

To the solutions of a diophantine equation with $r$ unknowns correspond geometrically - as we know - in the $r$-dimensional space $R^{r}$ the points with entire coordinates of an $(r-1)$-dimensional hypersurface. From this geometrical interpretation follows immediately for every diophantine equation with $r$ unknowns the following problem of a very general character, which can be formulated also merely arithmetically: how the lattice points representing the solutions of the diophantine equation in question wre distributed in the space $R^{r}$. Of course this problem is interesting principally in the case when the diophantine equation has infinitely many solutions.

Let $r$ and $P$ be positive integers, $\Phi\left(x_{1}, \ldots, x_{r}\right)$ a polynomial of $r$ variables with entire coefficients, in respect to which we do not make, for the moment, any restrictions.

The distribution of the solutions in positive integers of the equation

$$
\Phi\left(x_{1}, \ldots, x_{r}\right)=0
$$

can be described with the aid of the solution function $R(P)$ defined in the following manner: let $R(P)$ denote the number of all the points with entire coordinates of the hypersurface (1) which are placed inside the cube $1 \leqslant x_{1} \leqslant P, \ldots, 1 \leqslant x_{r} \leqslant P$.

Purely arithmetically formulated, $R(P)$ means the number of all the positive entire solutions of the diophantine equation (1) in respect to which $x_{1} \leqslant P, \ldots, x_{r} \leqslant P$.

As each of the variables $x_{1}, \ldots, x_{r}$ can assume only the values $1, \ldots, P$, for the $R(P)$ solution function we have in every case the trivial upper estimation

$$
R(P) \leqslant P^{r} \text {. }
$$

But in very many cases the upper estimation can be considerably improved. So for instance if $n \geqslant 2$, and $O=C(\varepsilon, n)$ is a positive constant de- 